PROCEEDINGS OF THE

AMERICAN MATHEMATICAL SOCIETY

Volume 128, Number 4, Pages 1215-1219

S 0002-9939(99)05119-9

Article electronically published on August 3, 1999

\title{
ALL NON-P-POINTS ARE THE LIMITS OF NONTRIVIAL SEQUENCES IN SUPERCOMPACT SPACES
}

\author{
ZHONGQIANG YANG AND WEI SUN
}

(Communicated by Alan Dow)

\begin{abstract}
A Hausdorff topological space is called supercompact if there exists a subbase such that every cover consisting of this subbase has a subcover consisting of two elements. In this paper, we prove that every non-P-point in any continuous image of a supercompact space is the limit of a nontrivial sequence. We also prove that every non-P-point in a closed $G_{\delta}$-subspace of a supercompact space is a cluster point of a subset with cardinal number $\leq c$. But we do not know whether this statement holds when replacing $c$ by the countable cardinal number. As an application, we prove in ZFC that there exists a countable stratifiable space which has no supercompact compactification.
\end{abstract}

\section{INTRODUCTION}

In this paper, all spaces are assumed to be Hausdorff topological spaces. The notation of supercompactness was introduced by de Groot [6]. A space $X$ is called supercompact if there exists a subbase $\mathcal{S}$ for $X$ such that every cover of $X$ consisting of elements of $\mathcal{S}$ has a subcover consisting of two elements. By the Alexander subbase lemma (we recently gave a simple proof for this lemma [12]), every supercompact space is compact. All continuous images of linearly ordered compacta are supercompact [2]. But the Cech-Stone compactification $\beta \omega$ of the infinite countable discrete space $\omega$ is not supercompact (see [1] or Section 3 in the present paper). In a space $X$ a point $p$ is called a $P$-point if $x \notin(\bigcup \mathcal{C})^{-} \backslash \cup \mathcal{C}$ for any countable family $\mathcal{C}$ of closed subsets of $X$; a point $p$ is called a weak P-point if $x \notin C^{-} \backslash C$ for any countable subset $C$ of $X$. It is trivial that every P-point is a weak P-point. However, there exists a non-P-point weak P-point in $\beta \omega \backslash \omega$ 8. In 1994, the first author of this paper in [11] proved that in a continuous image of a closed $G_{\delta^{-}}$-subspace of a supercompact space every non-weak-P-point is the limit of a nontrivial sequence and answered some problems in [4] and [9]. In the present paper, we prove the following theorems:

Theorem 1. Let $Y$ be a continuous image of a supercompact space and y a non$P$-point in $Y$. Then $y$ is the limit of a nontrivial sequence in $Y$.

Received by the editors March 8, 1998 and, in revised form, May 20, 1998.

1991 Mathematics Subject Classification. Primary 54D30.

Key words and phrases. Supercompact, P-point, sequence, compactification.

This work is supported by the National Education Committee of China for outstanding youths and by the National Education Committee of China for Scholars returning from abroad. 
Theorem 2. Let $Y$ be a closed $G_{\delta}$-subspace of a supercompact space and y a non$P$-point in $Y$. Then there exists a subset $A$ of $Y$ such that $p \in A^{-} \backslash A$ and $|A| \leq c$, where $c$ is the cardinal number of the set of all real numbers.

Thus we propose the following problem:

Problem 1. Under the assumptions of Theorem 2, we ask if there must be a countable subset $A$ of $Y$ such that $p$ is a cluster point of $A$. That is, are P-point and weak-P-point equivalent in any closed $G_{\delta}$-subspace of a supercompact space?

Remark 1. The statement in Theorem 2 does not hold for any compact Hausdorff space. In fact, Theorem 3.2 and Proposition 4.8 in Dow [5] imply that for any cardinal number $\kappa$ there exists a compact Hausdorff space $X$ such that $X$ contains a non-P-point which is not a cluster point of any set in $X$ with size at most $\kappa .1$

\section{Proofs of the MAIN THEOREMS}

Now we give proofs of the above theorems. At first, let us list some notation. Let $\mathcal{S}$ be a family of subsets in a topological space $X$. If the family $\{X \backslash S: S \in \mathcal{S}\}$ is a subbase for $X$, then $\mathcal{S}$ is called a closed subbase for $X$. If every pair of elements of $\mathcal{S}$ has a nonempty intersection, then $\mathcal{S}$ is called linked. If every linked subfamily of $\mathcal{S}$ has a nonempty intersection, then $\mathcal{S}$ is called binary. Obviously, a space is supercompact if and only if it has a binary closed subbase. Furthermore, we can assume that this closed subbase is closed with respect to arbitrary intersection. The following lemma proved in [11] is necessary to prove our theorems.

Lemma 1. Let $\mathcal{S}$ be a closed subbase for a compact space $X$ which is closed with arbitrary intersection, $F$ a closed set and $U$ an open set in $X$ with $F \subset U$. Then there exists a finite subfamily $\mathcal{F}$ of $\mathcal{S}$ such that $F \subset$ int $(\bigcup \mathcal{F}) \subset \bigcup \mathcal{F} \subset U$. Furthermore, if $F=\{p\}$ is a single point set, then $\mathcal{F}$ satisfies also that $p \in \cap \mathcal{F}$.

Proof of Theorem 1. Let $X$ be a supercompact space with a binary closed subbase $\mathcal{S}$ which is closed with respect to arbitrary intersection and $X \in \mathcal{S}$. Let $f: X \longrightarrow Y$ be a continuous mapping from $X$ onto $Y$. Suppose $\mathcal{B}$ is a countable family of closed sets of $Y$ such that

$$
y \in(\bigcup \mathcal{B})^{-} \backslash \bigcup \mathcal{B}
$$

Let $\mathcal{A}=\left\{f^{-1}(B): B \in \mathcal{B}\right\}$. Then there exists $p \in f^{-1}(y)$ such that $p \in$ $(\bigcup \mathcal{A})^{-} \backslash \bigcup \mathcal{A}$ because $f$ is a closed mapping. By Lemma 1 , for every $A \in \mathcal{A}$, there exists a finite subfamily $\mathcal{S}(A)$ of $\mathcal{S}$ such that $A \subset \bigcup \mathcal{S}(A) \subset X \backslash f^{-1}(y)$. Let $\mathcal{F}=$ $\bigcup\{\mathcal{S}(A): A \in \mathcal{A}\}$. Then $\mathcal{F}$ is a countable subfamily of $\mathcal{S}$ and $p \in(\bigcup \mathcal{F})^{-} \backslash \bigcup \mathcal{F}$. Now for every $F \in \mathcal{F}$, the family

$$
\{F\} \cup\{S \in \mathcal{S}: S \cap F \neq \emptyset \text { and } p \in S\}
$$

is a linked subfamily of $\mathcal{S}$ and hence it has a nonempty intersection. Choose a point $x_{F}$ in this intersection and let $C=\left\{x_{F}: F \in \mathcal{F}\right\}$. Then $C$ is a countable set of $X$ and $f^{-1}(y) \cap C=\emptyset$. In order to prove $y$ is a cluster point of the countable set $f(C)$, it remains to verify that $p \in C^{-}$. In fact, if $p \notin C^{-}$, then, by Lemma 1 , there exists a finite subfamily $\mathcal{S}_{0}$ of $\mathcal{S}$ such that

$$
p \in \operatorname{int}\left(\bigcup \mathcal{S}_{0}\right) \cap \bigcap \mathcal{S}_{0} \subset \bigcup \mathcal{S}_{0} \subset X \backslash C^{-} .
$$

\footnotetext{
${ }^{1}$ This remark is due to Professor M. G. Bell in University of Manitoba (Canada).
} 
Because $\bigcup \mathcal{S}_{0}$ is a neighborhood of $p$ and $p \in(\bigcup \mathcal{F})^{-} \backslash \bigcup \mathcal{F}$, there exists $F \in \mathcal{F}$ such that $\bigcup \mathcal{S}_{0} \cap F \neq \emptyset$. Hence there exists $S \in \mathcal{S}_{0}$ such that $F \cap S \neq \emptyset$. It follows from the definition of $x_{F}$ that $x_{F} \in S$. This contradicts with (1). Thus we have proved that $y$ is not a weak-P-point in $Y$. It follows from the theorem in [11] that $y$ is the limit of a nontrivial sequence in $Y$.

Proof of Theorem 2. Let $X$ be a supercompact space with a binary closed subbase $\mathcal{S}$ which is closed with respect to arbitrary intersection and $X \in \mathcal{S}$. Let $Y \subset X$ be a closed $G_{\delta}$-subspace of $X$. Then there exists a sequence $\left\{U_{1}, U_{2}, \cdots\right\}$ of open sets of $X$ such that $U_{1} \supset U_{2} \supset \cdots$ and $\bigcap_{n=1}^{\infty} U_{n}=Y$. Since $y$ is not a P-point in $Y$, there exists a countable family $\mathcal{C}$ of closed sets in $Y$ (hence in $X$ ) such that $y \in(\bigcup \mathcal{C})^{-} \backslash \bigcup \mathcal{C}$. Now for every $n$ and $C \in \mathcal{C}$, by Lemma 1 , there exists a finite subfamily $\mathcal{S}(C, n)$ of $\mathcal{S}$ such that

$$
C \subset \bigcup \mathcal{S}(C, n) \subset U_{n} \backslash\{y\} .
$$

Hence,

$$
C \subset \bigcap_{n=1}^{\infty} \bigcup \mathcal{S}(C, n)=\bigcup\left\{\bigcap_{n=1}^{\infty} f(n): f \in \prod_{n=1}^{\infty} \mathcal{S}(C, n)\right\} .
$$

For every $f \in \prod_{n=1}^{\infty} \mathcal{S}(C, n)$, let

$$
S(C, f)=\bigcap_{n=1}^{\infty} f(n) .
$$

Then $S(C, f) \subset \bigcap_{n=1}^{\infty}\left(U_{n} \backslash\{y\}\right)=Y \backslash\{y\}$ and $S(C, f) \in \mathcal{S}$ since $\mathcal{S}$ is closed with respect to arbitrary intersection. Furthermore,

$$
C \subset \bigcup\left\{S(C, f): f \in \prod_{n=1}^{\infty} \mathcal{S}(C, n)\right\} .
$$

Thus,

$$
y \in\left(\bigcup\left\{S(C, f): C \in \mathcal{C} \text { and } f \in \prod_{n=1}^{\infty} \mathcal{S}(C, n)\right\}\right)^{-} .
$$

Hence, similar to Theorem 1, we may choose $x(C, f) \in S(C, f)$ satisfying that $y$ is a cluster point of the set $A$ of all $x(C, f)$ 's. It is trivial that $|A| \leq c$. Thus we complete the proof of Theorem 2.

Remark 2. It is not difficult to extend our theorems from the countable cardinal number to any cardinal number.

\section{An APPLICATION}

It is an important topic to give some classes of Tychonoff spaces having supercompact compactifications. All separable metrizable spaces have supercompact compactifications since all compact metrizable spaces are supercompact [3. But it seem to be yet open whether all metrizable spaces have supercompact compactifications [7]. Van Mill [7] proved that if $p \in \beta \omega \backslash \omega$ is a P-point in $\beta \omega \backslash \omega$, then the space $\omega \cup\{p\}$ has no supercompact compactification. However, S. Shelah proved that the existence of a P-point in $\beta \omega \backslash \omega$ is only a consistent result but not a theorem in ZFC (see [10]). Thus van Mill's theorem cannot imply in ZFC that there exists a stratifiable space having no supercompact compactification. Applying Theorem 1 in the present paper we, however, can obtain many countable stratifiable spaces 
which have no supercompact compactification. In particular, the space $\omega \cup\{p\}$ has no supercompact compactification for every $p \in \beta \omega \backslash \omega$.

The following simple lemma seems to be known:

Lemma 2. Let $X$ be a Tychonoff space and $p \in \beta X \backslash X$. Then for every compactification $\gamma(X \cup\{p\})$ of the space $X \cup\{p\} \subset \beta X, p$ is the limit of a nontrivial sequence in $\gamma(X \cup\{p\})$ if and only if so is $p$ in $\beta X$.

Proof. It suffices to verify the following fact:

For any compactification $\gamma(X \cup\{p\})$ of the space $X \cup\{p\}$ and the unique extension $f: \beta X=\beta(X \cup\{p\}) \longrightarrow \gamma(X \cup\{p\})$ of the embedding $i: X \cup\{p\} \longrightarrow \gamma(X \cup\{p\})$ we have $f^{-1}(p)=\{p\}$.

In fact, if $f(q)=p$ for some $q \in \beta X$ but $q \neq p$, then there exist open sets $U, V \subset \beta X$ such that $p \in U, q \in V$ and $U_{\beta X}^{-} \cap V_{\beta X}^{-}=\emptyset$. It follows that

$$
p \in f\left(V_{\beta X}^{-}\right)=f\left((V \cap X)_{\beta X}^{-}\right)=(f(V \cap X))_{\gamma(X \cup\{p\})}^{-}=(V \cap X)_{\gamma(X \cup\{p\}}^{-} .
$$

Thus

$$
p \in(V \cap X)_{\gamma(X \cup\{p\})}^{-} \cap(X \cup\{p\})=(V \cap X)_{X \cup\{p\}}^{-} \subset(V \cap X)_{\beta X}^{-}=V_{\beta X}^{-} .
$$

A contradiction occurs.

Theorem 3. Let $X$ be a Tychonoff space with a dense subset which may be represented as a union of countably many compact sets. If $p \in \beta X \backslash X$ is not the limit of any nontrivial sequence in $\beta X$, then there exists no supercompact compactification of the space $X \cup\{p\}$.

Proof. It follows from Theorem 1 and Lemma 2 since $p$ is not a P-point in any compactification of the space $X \cup\{p\}$.

Corollary 1. There exists a countable space with only one nonisolated point having no supercompact compactification.

Proof. $\omega \cup\{p\}$ is such a space for every $p \in \beta \omega \backslash \omega$.

\section{ACKNOWLedgement}

The authors would like to thank the referee and the editor for their valuable suggestions for the improvement of the paper.

\section{REFERENCES}

[1] Bell M. G., Not all compact Hausdorff spaces are supercompact, General Topology Appl. 8(1978), pp. 151-155.

[2] Bula W., Nikiel J., Tuncali H. M. and Tymchatyn E.D., Continuous images of ordered compacta are regular supercompact, Topology Appl. 45(1992), pp. 203-221. [MR 93i:54015

[3] van Douwen E. K., Special bases for compact metrizable spaces, Fund. Math. 61(1981), pp. 201-209. MR 82d:54036

[4] van Douwen E. K. and van Mill J., Supercompact spaces, Topology Appl. 1(1982), pp. 21-32. MR 82m:54017

[5] Dow A., Good and OK ultrafilters, Trans. Amer. Math. Soc. 290(1985), pp. 145-160. MR 86f:54044

[6] de Groot J., Supercompactness and superextension, Contribution to Extension Theory of Topological Structures, Proceedings 1967 Berlin Symposium, Berlin(1969), pp. 89-90.

[7] van Mill J., A countable space no compactification of which is supercompact, Bull. Acad. Pol. Sci. 25(1972), pp. 1129-1132. MR 58:2719 
[8] van Mill J., A introduction to $\beta \omega$, Handbook of Set-Theoretic Topology (ed. by Kunen K. and Vaughan J.E.), pp. 503-568. MR 86f:54027

[9] van Mill J. and Mills C. F., Closed $G_{\delta}$-subsets of supercompact Hausdorff spaces, Indag. Math. (N.S.) 41(1979), pp. 155-162. IMR 80e:54026

[10] Wimmers E. L., The Shelah P-point independence theorem, Israel J. Math. 43(1982), pp. 2848. MR 85e:03118

[11] Yang Zhongqiang, All cluster points of countable sets in supercompact spaces are the limits of nontrivial sequences, Proc. Amer. Math. Soc. 122(1994), pp. 591-595. MR 95a:54041

[12] Yang Zhongqiang, A simple proof for the Alexander subbase lemma, J. Shaanxi Normal University 26(1998), pp. 23-26 (In Chinese).

Department of Mathematics, Shaanxi Normal University, Xi'an, 710062, People's RePUBLIC OF CHINA

E-mail address: yangmathsnuc@ihw.com.cn

Xi'an Institute of Technology, Xi'an, 710032, People's Republic of China 